\title{
Progranulin deficiency promotes neuroinflammation and neuron loss following toxin-induced injury
}

\author{
Lauren Herl Martens, ${ }^{1}$ Jiasheng Zhang,, ${ }^{2,3}$ Sami J. Barmada, ${ }^{4,5}$ Ping Zhou, ${ }^{1}$ \\ Sherry Kamiya, ${ }^{2,3}$ Binggui Sun, ${ }^{4}$ Sang-Won Min, ${ }^{4}$ Li Gan, ${ }^{4,6}$ Steven Finkbeiner, 4,5,6,7,8 \\ Eric J. Huang, ${ }^{2,3,6}$ and Robert V. Farese Jr.1,6,9
}

\begin{abstract}
${ }^{1}$ Gladstone Institute of Cardiovascular Disease, San Francisco, California, USA. ${ }^{2}$ Department of Pathology, UCSF, San Francisco, California, USA. ${ }^{3}$ Pathology Service 113B, VA Medical Center, San Francisco, California, USA. ${ }^{4}$ Gladstone Institute of Neurodegenerative Disease, San Francisco, California, USA. ${ }^{5}$ Department of Neurology, ${ }^{6}$ Consortium for Frontotemporal Dementia Research, ${ }^{7}$ Department of Physiology, ${ }^{8}$ Taube-Koret Center for Huntington's Disease Research and Hellman Family Foundation Program in Alzheimer's Disease Research, and ${ }^{9}$ Departments of Medicine, Biochemistry and Biophysics, UCSF, San Francisco, California, USA.
\end{abstract}

\begin{abstract}
Progranulin (PGRN) is a widely expressed secreted protein that is linked to inflammation. In humans, PGRN haploinsufficiency is a major inherited cause of frontotemporal dementia (FTD), but how PGRN deficiency causes neurodegeneration is unknown. Here we show that loss of PGRN results in increased neuron loss in response to injury in the CNS. When exposed acutely to 1-methyl-4-(2'-methylphenyl)-1,2,3,6-tetrahydrophine (MPTP), mice lacking PGRN $\left(\mathrm{Grn}^{-/-}\right)$showed more neuron loss and increased microgliosis compared with wild-type mice. The exacerbated neuron loss was due not to selective vulnerability of $\mathrm{Grn}^{-/-}$neurons to MPTP, but rather to an increased microglial inflammatory response. Consistent with this, conditional mutants lacking PGRN in microglia exhibited MPTP-induced phenotypes similar to $\mathrm{Grn}^{-/-}$mice. Selective depletion of PGRN from microglia in mixed cortical cultures resulted in increased death of wild-type neurons in the absence of injury. Furthermore, $\mathrm{Grn}^{-/-}$microglia treated with LPS/IFN- $\gamma$ exhibited an amplified inflammatory response, and conditioned media from these microglia promoted death of cultured neurons. Our results indicate that PGRN deficiency leads to dysregulated microglial activation and thereby contributes to increased neuron loss with injury. These findings suggest that PGRN deficiency may cause increased neuron loss in other forms of CNS injury accompanied by neuroinflammation.
\end{abstract}

\section{Introduction}

Progranulin (PGRN) is an approximately $70-\mathrm{kDa}$ secreted protein involved in cellular processes such as wound healing and inflammation $(1,2)$. PGRN has been implicated as a regulator of TNF- $\alpha-$ mediated inflammation (3) and also has growth factor properties, with roles in cellular proliferation and survival (1). PGRN can be processed to granulin (GRN) peptides (4), which may have different functions (5). PGRN is expressed widely in tissues and circulates in the blood and cerebrospinal fluid $(6,7)$.

Mutations in GRN are causally linked to frontotemporal dementia (FTD) (8-10). Various types of mutations have been found that result in GRN haploinsufficiency and cause a $>50 \%$ reduction in circulating PGRN (11). PGRN is expressed in neurons and microglia (7); the contribution of PGRN deficiency in these cell types to FTD is unknown. Intracellular aggregates of TDP-43 are found in neurons of subjects with PGRN-deficient FTD, and TDP43 may contribute to neuron loss (12). However, microglial PGRN expression is increased in Alzheimer's disease and amyotrophic lateral sclerosis $(13,14)$, suggesting that it may play a more general role in neuroinflammation and neurodegeneration.

We and several groups have used murine models to examine the effects of PGRN deficiency in the CNS. Neurons of PGRNknockout $\left(\mathrm{Grn}^{-/-}\right)$mice develop ubiquitin-positive aggregates and phosphorylated TDP-43, similar to FTD patients with GRN mutations (15-20). Neurons from $\mathrm{Grn}^{-/-}$mice exhibit

Conflict of interest: The authors have declared that no conflict of interest exists. Citation for this article: J Clin Invest. 2012;122(11):3955-3959. doi:10.1172/JCI63113. reduced survival in culture (21). In addition, PGRN deficiency results in gliosis in the CNS of aged mice (L.H. Martens, unpublished observations, and refs. 15-20). PGRN expression is also upregulated following axotomy of the sciatic nerve in the peripheral nervous system (22). These latter observations suggest that PGRN may modulate inflammation in the CNS, consistent with studies showing that PGRN deficiency predisposes macrophages to increased inflammation (15).

Here we tested the hypothesis that PGRN attenuates the inflammatory response to CNS injury. We used the acute injury model of treatment with 1-methyl-4-(2'-methylphenyl)-1,2,3,6tetrahydrophine (MPTP), a neurotoxin that targets the dopaminergic neurons of the substantia nigra pars compacta ( $\mathrm{SNpc}$ ). Although MPTP injury is commonly used as a Parkinson's disease model, it provides a reproducible CNS injury with consistent neuron loss and reactive gliosis (23). We examined the effects of MPTP on neuron survival and microglial activation in PGRN-deficient mice and utilized primary cultures of neurons and microglia to investigate mechanisms.

\section{Results and Discussion}

To investigate PGRN expression and function in the CNS, we generated a conditional $\mathrm{Gm}$ allele and, subsequently, $\mathrm{Grm}^{-/}$mice by crossing $G m^{\text {flfl }}$ mice with $\beta$-actin-Cre mice (ref. 24 and Supplemental Figure 1, A-C; supplemental material available online with this article; doi:10.1172/JCI63113DS1). In adult mouse brains, PGRN was expressed primarily in neurons and in resting and reactive microglia (Supplemental Figure 1D and Supplemental Figure 2, A-C). 
A
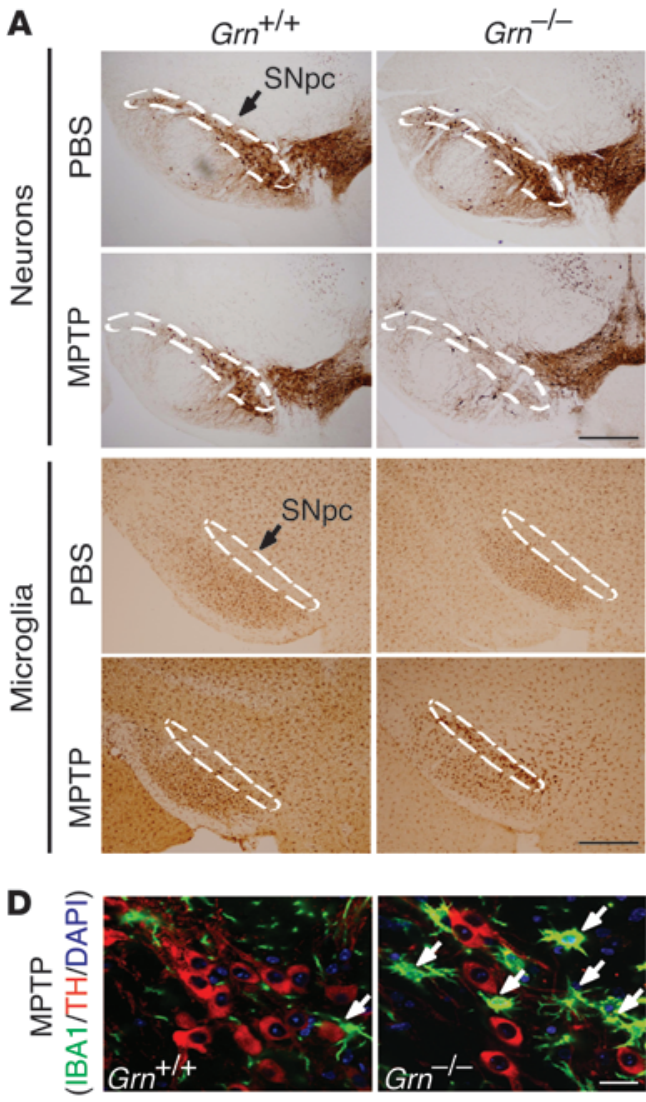

B
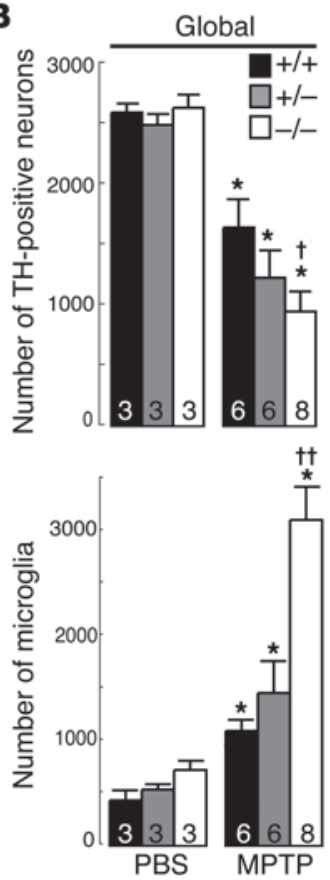

C
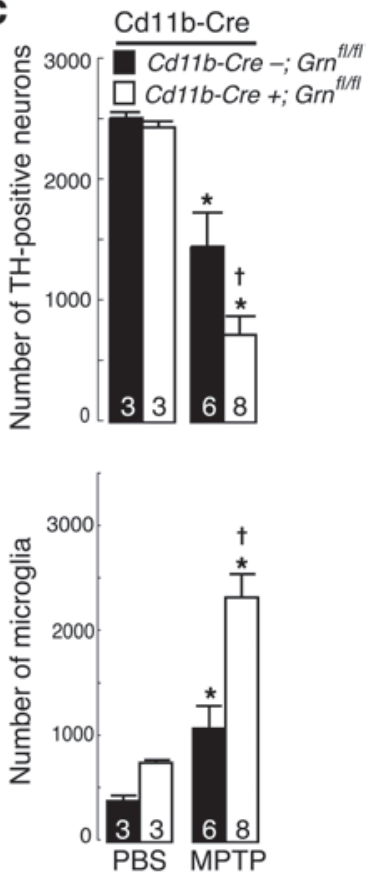

Figure 1

Increased neuron death and microglial activation caused by PGRN deficiency in a model of CNS injury. (A) Representative SNpc sections showing decreased numbers of TH-positive neurons and increased numbers of IBA1-positive microglia following MPTP exposure in PGRN-deficient mice. Dashed lines indicate SNpc. Scale bars: $500 \mu \mathrm{m}$. (B) Quantification of TH-positive neurons and IBA1-positive microglia in the SNpc in the global PGRNdeficient mouse model. Numbers denote $n$ per group. ${ }^{\star} P<0.05$, MPTP versus PBS; ${ }^{\dagger} P<0.05$, Grn ${ }^{+/+}$MPTP versus Grn ${ }^{-1-}$ MPTP; ${ }^{\dagger+} P<0.01$, Grn ${ }^{-/-}$ MPTP versus either $\mathrm{Grn}^{+/+}$or Grn+/- MPTP. (C) Quantification of TH-positive and IBA1-positive microglia in the SNpc in the microglia-targeted PGRNdeficient mouse model. Numbers denote $n$ per group. ${ }^{*} P<0.05$, MPTP versus PBS; ${ }^{\top} P<0.05, C d 11 b$-Cre;Grn ${ }^{f l / f l}$ MPTP versus Cd11b-Cre+;Grn ${ }^{f l / f l}$ MPTP. (D) Representative confocal images showing that MPTP treatment decreased the number of TH-positive neurons (red) and increased the numbers of activated microglia (green) in the SNpc of $\mathrm{Grn}^{-/-}$mice. Arrows indicate activated microglia. Scale bar: $20 \mu \mathrm{m}$.

Because PGRN is expressed in activated microglia (22) and antagonizes inflammation (3), we hypothesized that PGRN attenuates the inflammatory response to CNS injury. To test this hypothesis, we chose a model in which injury was induced by MPTP, which, at high doses, acutely causes inflammation and death of SNpc dopaminergic neurons (23). Neuron toxicity is in part due to conversion of MPTP to $\mathrm{MPP}^{+}$, which is taken up by dopaminergic neurons and blocks electron transport (23). Additionally, microglia are activated and secrete cytotoxic, proinflammatory cytokines, such as TNF- $\alpha(23,25)$.

Three-month-old Grn+/+, Grn+/-, and $\mathrm{Grn}^{-/-}$mice were treated with MPTP or vehicle (PBS) for 2 days (Figure 1A). In vehicle-treated mice of any genotype, the numbers of dopaminergic (tyrosine hydroxylase $[\mathrm{TH}]$-positive) neurons in SNpc were similar, indicating that PGRN deficiency did not affect their survival (Figure 1, A and B). As expected, MPTP treatment induced approximately $37 \%$ loss of dopaminergic neurons in the SNpc of $\mathrm{Grn}^{+/+}$mice (Figure 1B). More strikingly, dopaminergic neurons were reduced by approximately $66 \%$ in $\mathrm{Grn}^{-/-}$mice, and by approximately $50 \%$ in $\mathrm{Grn}^{+/-}$mice. Quantification of Nissl-positive neurons in SNpc showed similar deficits in $\mathrm{Grn}^{+/+}$and $\mathrm{Grn}^{-/-}$mice following MPTP treatment (Supplemental Figure 3, A and B). While there were no detectable differences in the numbers of resting microglia, MPTP treatment increased activated microglia in SNpc by 2.84 -fold in $\mathrm{Grn}^{-/-}$mice and 1.33-fold in $\mathrm{Grn}^{+/-}$mice, compared with $\mathrm{Grn}^{+/+}$mice (Figure 1, B and D). Most Grn-/- microglia were highly ramified and large, indicative of activated microglia (Figure 1D and ref. 25).

To further investigate the contribution of PGRN expression in microglia in the MPTP-induced injury model, we crossed Grn ${ }^{f / f l}$ mice with Cd11b-Cre mice (26) to generate mice with PGRN deficiency in microglia (Cd11b-Cre+;Grn fl/fl). PGRN mRNA levels and secretion were decreased in primary microglia cultured from Cd11b-Cre+; Gr $n^{f / f l}$ mice by approximately $54 \%$ and $42 \%$, respectively, but not cortical neurons (Supplemental Figure 4, A and B). Consistent with the results with $\mathrm{Grn}^{-/-}$mice, $\mathrm{Cd} 11 \mathrm{~b}-\mathrm{Cre}+; \mathrm{Grn}^{f / f l}$ mice showed a similar reduction in dopaminergic neurons (Figure 1C and Supplemental Figure 4C) and increased microgliosis after MPTP treatment (Figure 1C and Supplemental Figure 4D). Together, these results supported that PGRN deficiency in microglia is sufficient to cause increased inflammation and reduced neuron survival following CNS injury. 


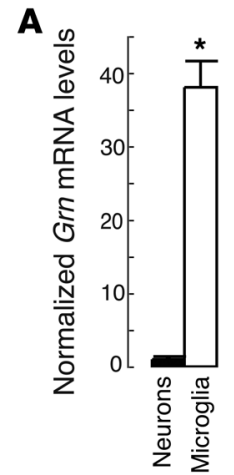

D

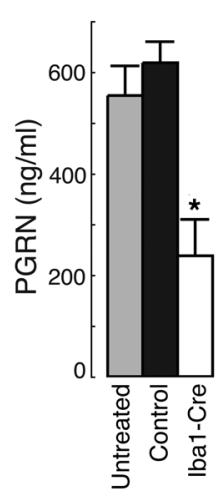

B

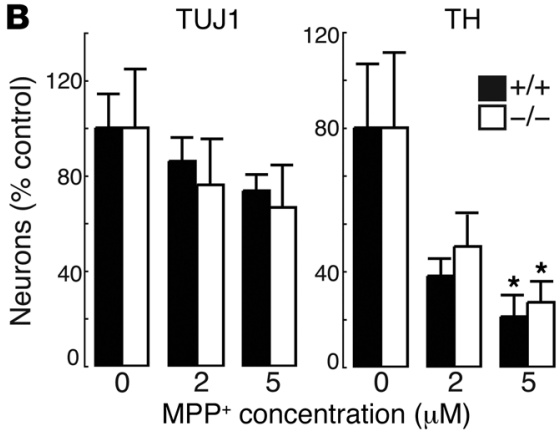

$\mathbf{E}$

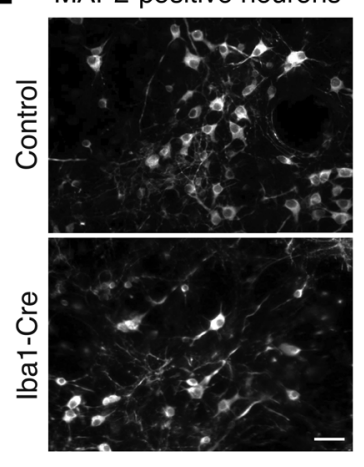

C

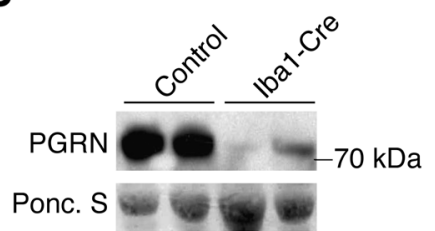

\section{Figure 2}

Microglial PGRN deficiency increases the death of cultured neurons. (A) Grn mRNA levels in cultured primary neurons and microglia. ${ }^{*} P<0.001$. (B) Similar numbers of surviving $\mathrm{Grn}^{+/+}$and $\mathrm{Grn}^{-/-}$midbrain neurons (TUJ1 or TH immunolabeled) following MPP+ treatment. ${ }^{*} P<0.05,5 \mu \mathrm{M}$ versus untreated. (C) Immunoblot showing reduced levels of secreted PGRN in Grntl/fl mixed cortical cultures infected with Iba1-Cre lentivirus. (D) Quantification of secreted PGRN in mixed cortical cultures. ${ }^{*} P<0.01$. (E) Representative image showing decreased survival of MAP2-positive neurons in mixed cultures lacking microglial PGRN compared with control cultures. ${ }^{\star} P<0.001$. Scale bar: $20 \mu \mathrm{m}$. (F) Increased risk of death for wild-type neurons exposed to conditioned media from LPS/IFN- $\gamma$-treated PGRN-deficient microglia. ${ }^{*} P<0.0001$ (log-rank test), LPS/IFN- $\gamma-$ treated conditioned media compared with PBS-treated conditioned media.

To further investigate the effects of PGRN deficiency in microglia, we utilized primary cell culture models. Both primary neurons and microglia expressed Grn mRNA, with much higher levels in microglia (Figure 2A), which are activated during culturing (27). We examined whether PGRN deficiency alters neuron survival in the absence of glia by testing the susceptibility of $\mathrm{Grn}^{+/+}$ and $\mathrm{Grn}^{-/-}$dopaminergic neurons (E13.5) to various doses of $\mathrm{MPP}^{+}$ for 3 days. Neuron death occurred in a dose-dependent manner following $\mathrm{MPP}^{+}$exposure, but there were no differences in the numbers of surviving wild-type or PGRN-deficient TH- and TUJ1positive neurons (Figure $2 \mathrm{~B}$ ).

We next asked whether PGRN deficiency in microglia had effects on neuron survival. We isolated mixed cortical cultures - containing neurons, microglia, and astrocytes - from (P0) $G r n^{f l / f l}$ mice and selectively depleted Grn in microglia by infecting with lentivirus expressing Iba1-Cre recombinase (Iba1Cre). Depletion of PGRN from microglia reduced PGRN in the medium by approximately $70 \%$ (Figure 2, C and D) and reduced the survival of MAP2-positive neurons by $>50 \%$ compared with controls (Figure 2E). There were no apparent changes in the numbers of microglia or astrocytes (data not shown). Thus, PGRN deficiency in microglia is sufficient to increase death of wild-type neurons in primary cultures.
We hypothesized that the increased neuron death observed when microglia are deficient in PGRN may be attributable to alterations in secreted factors. To test this hypothesis, we collected conditioned media from $\mathrm{Grn}^{+/+}$and $\mathrm{Grn}^{-/-}$microglia treated with either PBS or LPS/IFN- $\gamma$ for 24 hours and then transferred the media to wild-type cortical neurons to assess survival. The cumulative risk of death for each treatment was determined using KaplanMeier survival analysis, which assessed the probability of neuron cell death over time $(28,29)$. There were no differences in survival of neurons exposed to conditioned media of PBS-treated $\mathrm{Grn}^{+/+}$or $\mathrm{Grn}^{-/-}$microglia (Figure 2F). In contrast, exposure of $\mathrm{Grn}^{+/+}$and $\mathrm{Grn}^{-/-}$microglia to LPS/IFN- $\gamma$ caused significantly more neuron cell death than PBS, and this effect was accentuated with conditioned media from activated $\mathrm{Grn}^{-/-}$microglia. These results indicate that activated PGRN-deficient microglia likely secrete factors that promote the death of wild-type neurons.

To investigate this phenomenon further, we examined the inflammatory response in primary microglia following 24 hours of treatment with LPS/IFN- $\gamma$. Similar to findings for PGRN-deficient macrophages $(3,15)$, Grn ${ }^{-/-}$microglia expressed and secreted increased amounts of the proinflammatory cytokines Tnfa, $I l 1 b$, and $I l 6$ compared with controls (Figure 3, A and B). Grn-1microglia also expressed less anti-inflammatory Il10 mRNA after 

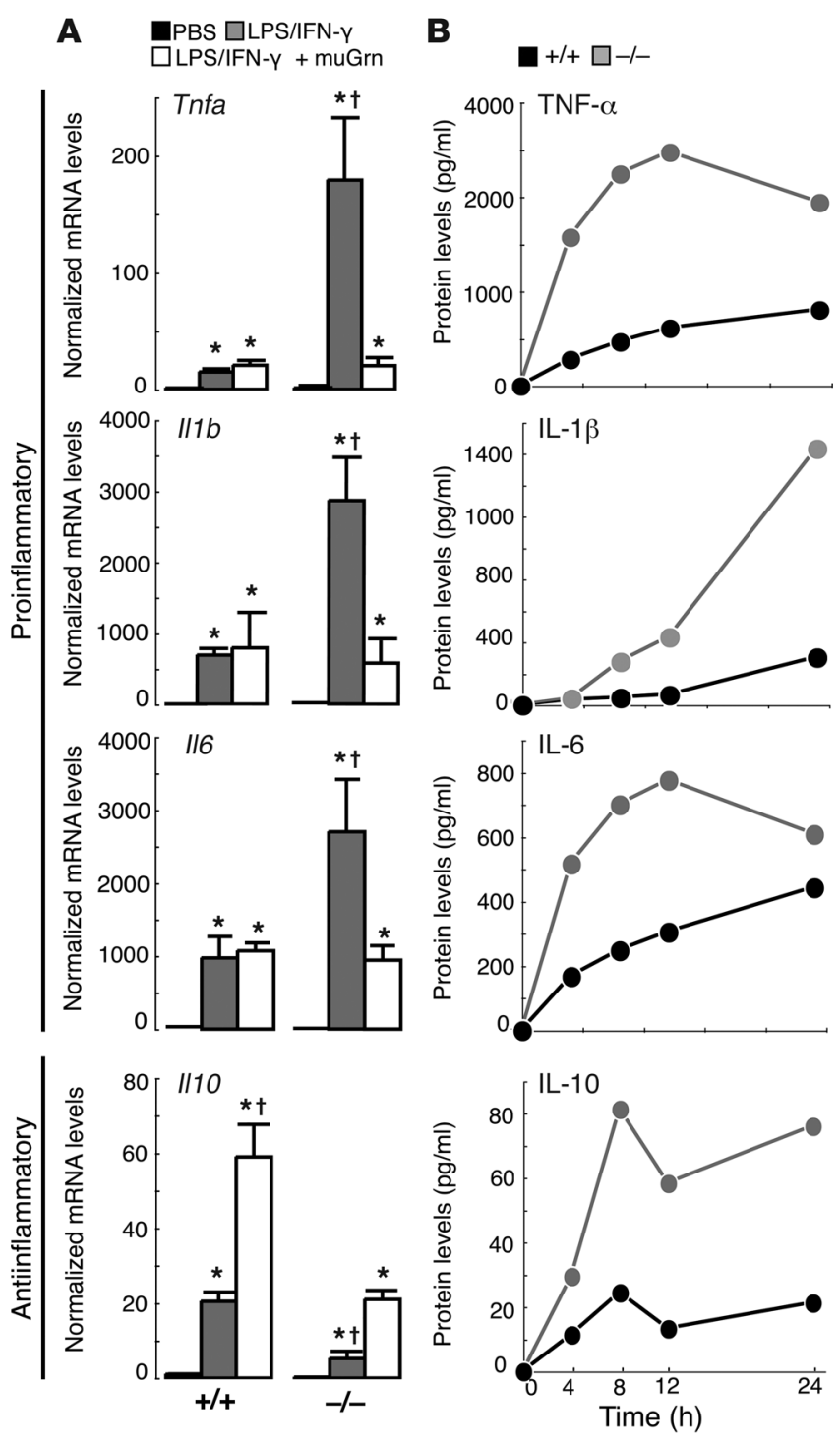

24 hours (Figure $3 \mathrm{~A}$ ), although increased amounts of IL-10 were secreted into the media (Figure $3 \mathrm{~B}$ ), reflecting differences in the regulation of mRNA versus secreted protein. The increased IL-10 secretion was apparently insufficient to dampen the increased inflammatory state of $\mathrm{Grn}^{-/-}$microglia. These data indicate that $\mathrm{Grn}^{-/-}$microglia are prone to a hyperactive proinflammatory state upon activation, and this hyperactivation likely contributes to the death of neurons. These data also suggest that a function of PGRN is to dampen the activation state of microglia upon stimulation. Supporting this, lentivirus-mediated overexpression of murine PGRN was sufficient to normalize cytokine mRNA levels in $\mathrm{Grn}^{-/-}$ microglia (Figure 3A and Supplemental Figure 5, A and B).

In this study, we tested the effects of PGRN deficiency on MPTP treatment, a well-established model of CNS injury that precipitates both neuron death and microglial activation (23). Our data indicate that PGRN deficiency results in an exaggerated, prolonged inflammatory response in activated microglia and that this mechanism likely contributes to enhanced neuron death following injury. We examined TNF- $\alpha$ as a potential mediator of this effect. TNF- $\alpha$ signaling contributes to the

\section{Figure 3}

PGRN-deficient microglia exhibit a hyperactivated inflammatory response. (A) Increased inflammatory cytokine mRNA levels in LPS/ IFN- $\gamma$-treated $\mathrm{Grn}^{-/-}$microglia, compared with $\mathrm{Grn}^{+/+}$controls, that was rescued by lentiviral infection with murine PGRN (MuGrn). ${ }^{*} P<0.05$ compared with PBS; ${ }^{\dagger} P<0.05$ compared with $\mathrm{Grn}^{-/-}$LPS/IFN- $\gamma$. (B) Representative time plots revealing increased secretion of inflammatory cytokines by LPS/IFN- $\gamma$-treated $\mathrm{Grn}^{-/-}$microglia compared with similarly treated $\mathrm{Grn}^{+/+}$cultures.

demise of SNpc dopaminergic neurons following MPTP treatment (30) and induces apoptosis in neurons (31). Additionally, mice lacking both TNFR1 and TNFR2 are protected against MPTP-induced dopaminergic neuron death (32). PGRN has been reported to bind TNF- $\alpha$ receptors and block signalling (3), suggesting that PGRN deficiency may lead to uncontrolled TNF- $\alpha$ signaling. However, we found that the addition of etanercept, soluble TNFR2, did not attenuate neuron death in our system (Supplemental Figure 6). We are unable to distinguish whether this was due to inadequate TNF- $\alpha$ blockade or whether other factors - such as increased cytokines or excitatory factors, or decreased trophic factors - are responsible for the increased neuron death.

Our findings suggest that PGRN deficiency may predispose microglia to hyperactivation and neuron death in FTD. Indeed, microglial activation is found in humans with FTD (33) and in murine models of PGRN deficiency (15-20). However, our findings do not exclude that PGRN deficiency in neurons may also be an important contributing factor. Several studies have demonstrated that PGRN has important neurotrophic properties $(21,34)$, and indeed, we found small reductions in the survival of $\mathrm{Grn}^{-/-}$cortical neurons cultured in nutrient-rich (B27) or nutrient-depleted (N2) media (Supplemental Table 1). Nevertheless, our results reveal a role for PGRN in attenuating neuroinflammation and suggest that this mechanism contributes to neurodegeneration in PGRN-deficient FTD. Further, PGRN may attenuate inflammation and neuron death in other forms of neurodegeneration or CNS injury. Therapies aimed at raising PGRN levels $(35,36)$ in the CNS therefore may prove useful as therapies for FTD and other forms of CNS injury.

\section{Methods}

Experimental procedures are described in detail in Supplemental Methods.

Generation of PGRN-deficient mice. A targeting vector was generated with loxP sites flanking the Grn coding sequence. Grn ${ }^{f / f l}$ mice were bred with mice expressing Cre recombinase under $\beta$-actin (24) or $C d 11 b$ (26) promoters.

Acute MPTP treatment. Three-month-old mice were treated with 4 i.p. injections of MPTP per day for 2 days ( $4 \mu \mathrm{g} / \mathrm{g}$ body weight) (Sigma-Aldrich) or PBS. Stereological counting was used to quantify dopamine neurons and microglia in the SNpc.

Primary cultures. Ventral midbrain, cortical neuron, mixed cortical, and microglial cultures were prepared from E13.5 or E16-18 embryos and P0 or $\mathrm{P} 2-\mathrm{P} 4$ pups.

Neuron survival assays. Pure cortical cultures were transfected with EGFP, microglial conditioned media was added, and cells were imaged every 24 hours using an automated microscopy system (28) to assess neuron death.

Cytokine analysis. Primary microglia were stimulated with PBS or $100 \mathrm{ng} / \mathrm{ml} \mathrm{LPS} \mathrm{(Sigma-Aldrich)} \mathrm{and} 100 \mathrm{U} / \mathrm{ml}$ IFN (Sigma-Aldrich). Secreted cytokine levels were measured using the Mouse Pro-inflammatory 7-plex cytokine ELISA kit (Meso Scale Discovery). 
Statistics. Results are mean \pm SEM unless otherwise stated. Differences between 2 groups were assessed with unpaired, 2-tailed $t$ tests. Comparisons involving more than 2 groups used an ANOVA with Tukey's post-hoc test. $P$ values less than 0.05 were considered significant. Tests were performed with InStat (GraphPad Software) or StatView software (SAS Institute Inc.); neuron survival analysis data utilized R (http://www.r-project.org/).

Study approval. All procedures were approved by the UCSF IACUC and followed the Guide for Care and Use of Laboratory Animals (NIH publication no. 85-23; revised 1985).

\section{Acknowledgments}

We thank L. Mitic and T. Nguyen for helpful discussions, L. Elia for murine PGRN ELISA assistance, Y. Zhou for lentivirus preparation, M.G. Tansey for etanercept, and G. Diaz-Ramirez for mouse husbandry. This work was supported by grants from the NIH (P50 AG023501 [to R.V. Farese Jr.], GMO 100909 [to R.V. Farese Jr.], F31 AG034793 [to L.H. Martens], P01 AG022074 [to S. Finkbeiner],
K26 OD010927 [to E.J. Huang], K08 NS072233 [SJB]), VA Merit Award BX001108 (to E.J. Huang), the Consortium for Frontotemporal Dementia Research (to R.V. Farese Jr.), the ALS Association (to S. Finkbeiner), and the Gladstone Institutes. The Gladstone Institutes received support from a National Center for Research Resources grant RR18928.

Received for publication January 30, 2012, and accepted in revised form August 17, 2012.

Address correspondence to: Eric J. Huang, Department of Pathology, University of California San Francisco, 513 Parnassus Avenue, San Francisco, California 94143-0502, USA. Phone: 415.476.8525; Fax: 415.514.0878; E-mail: eric.huang2@ucsf.edu. Or to: Robert V. Farese Jr., Gladstone Institutes, 1650 Owens Street, San Francisco, California 94158, USA. Phone: 415.734.2718; Fax: 415.355.0960; E-mail: bfarese@gladstone.ucsf.edu.
1. He Z, Ismail A, Kriazhev L, Sadvakassova G, Bateman A. Progranulin (PC-cell-derived growth factor/acrogranin) regulates invasion and cell survival. Cancer Res. 2002;62(19):5590-5596.

2. He Z, Ong CH, Halper J, Bateman A. Progranulin is a mediator of the wound response. Nat Med. 2003;9(2):225-229.

3. Tang $\mathrm{W}$, et al. The growth factor progranulin binds to TNF receptors and is therapeutic against inflammatory arthritis in mice. Science. 2011; 332(6028):478-484

4. Zhu J, et al. Conversion of proepithelin to epithelins: roles of SLPI and elastase in host defense and wound repair. Cell. 2002;111(6):867-878

5. Bateman A, Bennett HP. Granulins: the structure and function of an emerging family of growth factors. J Endocrinol. 1998;158(2):145-151.

6. Daniel R, He Z, Carmichael KP, Halper J, Bateman A. Cellular localization of gene expression for progranulin. J Histochem Cytochem. 2000;48(7):999-1009.

7. Petkau TL, et al. Progranulin expression in the developing and adult murine brain. J Comp Neurol. 2010;518(19):3931-3947.

8. Baker $\mathrm{M}$, et al. Mutations in progranulin cause tau-negative frontotemporal dementia linked to chromosome 17. Nature. 2006;442(7105):916-919.

9. Gass J, et al. Mutations in progranulin are a major cause of ubiquitin-positive frontotemporal lobar degeneration. Hum Mol Genet. 2006;15(20):2988-3001.

10. Cruts $\mathrm{M}$, et al. Null mutations in progranulin cause ubiquitin-positive frontotemporal dementia linked to chromosome 17q21. Nature. 2006;442(7105):920-924.

11. Finch $N$, et al. Plasma progranulin levels predict progranulin mutation status in frontotemporal dementia patients and asymptomatic family members. Brain. 2009;132(pt 3):583-591.

12. Neumann M, et al. Ubiquitinated TDP-43 in frontotemporal lobar degeneration and amyotrophic lateral sclerosis. Science. 2006;314(5796):130-133.

13. Irwin D, Lippa CF, Rosso A. Progranulin (PGRN) expression in ALS: an immunohistochemical study. J Neurol Sci. 2009;276(1-2):9-13.

14. Pereson S, et al. Progranulin expression corre- lates with dense-core amyloid plaque burden in Alzheimer disease mouse models. J Pathol. 2009; 219(2):173-181.

15. Yin F, et al. Exaggerated inflammation, impaired host defense, and neuropathology in progranulindeficient mice. J Exp Med. 2010;207(1):117-128.

16. Yin $\mathrm{F}$, et al. Behavioral deficits and progressive neuropathology in progranulin-deficient mice: a mouse model of frontotemporal dementia. FASEB J. 2010;24(12):4639-4647.

17. Wils $\mathrm{H}$, et al. Cellular ageing, increased mortality and FTLD-TDP-associated neuropathology in progranulin knockout mice. J Pathol. 2012; 228(1):67-76.

18. Petkau TL, et al. Synaptic dysfunction in progranulin-deficient mice. Neurobiol Dis. 2012;45(2):711-722.

19. Ahmed $Z$, et al. Accelerated lipofuscinosis and ubiquitination in granulin knockout mice suggest a role for progranulin in successful aging. Am J Pathol. 2010;177(1):311-324.

20. Ghoshal N, et al. Core features of frontotemporal dementia recapitulated in progranulin knockout mice. Neurobiol Dis. 2012;45(1):395-408.

21. Kleinberger $G$, et al. Increased caspase activation and decreased TDP-43 solubility in progranulin knockout cortical cultures. J Neurochem. 2010;115(3):735-747.

22. Moisse K, Volkening K, Leystra-Lantz C, Welch I, Hill T, Strong MJ. Divergent patterns of cytosolic TDP-43 and neuronal progranulin expression following axotomy: implications for TDP-43 in the physiological response to neuronal injury. Brain Res. 2009;1249:202-211.

23. Dauer W, Przedborski S. Parkinson's disease: mechanisms and models. Neuron. 2003;39(6):889-909.

24. Lewandoski M, Sun X, Martin GR. Fgf8 signalling from the AER is essential for normal limb development. Nat Genet. 2000;26(4):460-463.

25. Członkowska A, Kohutnicka M, Kurkowska-Jastrzebska I, Członkowski A. Microglial reaction in MPTP (1-methyl-4-phenyl-1,2,3,6-tetrahydropyridine) induced Parkinson's disease mice model. Neurodegeneration. 1996;5(2):137-143.

26 . Boillée $S$, et al. Onset and progression in inherited
ALS determined by motor neurons and microglia. Science. 2006;312(5778):1389-1392.

27. Ong $\mathrm{CH}$, et al. Regulation of progranulin expression in myeloid cells. Am J Physiol Regul Integr Comp Physiol. 2006;291(6):R1602-R1612.

28. Arrasate M, Finkbeiner S. Automated microscope system for determining factors that predict neuronal fate. Proc Natl Acad Sci US A. 2005;102(10):3840-3845.

29. Barmada SJ, Skibinski G, Korb E, Rao EJ, Wu JY, Finkbeiner S. Cytoplasmic mislocalization of TDP43 is toxic to neurons and enhanced by a mutation associated with familial amyotrophic lateral sclerosis. J Neurosci. 2010;30(2):639-649.

30. Ferger B, Leng A, Mura A, Hengerer B, Feldon J. Genetic ablation of tumor necrosis factor-alpha (TNF-alpha) and pharmacological inhibition of TNF-synthesis attenuates MPTP toxicity in mouse striatum. J Neurochem. 2004;89(4):822-833.

31. McGuire SO, Ling ZD, Lipton JW, Sortwell CE, Collier TJ, Carvey PM. Tumor necrosis factor alpha is toxic to embryonic mesencephalic dopamine neurons. Exp Neurol. 2001;169(2):219-230.

32. Sriram K, Matheson JM, Benkovic SA, Miller DB, Luster MI, O'Callaghan JP. Deficiency of TNF receptors suppresses microglial activation and alters the susceptibility of brain regions to MPTPinduced neurotoxicity: role of TNF-alpha. FASEB J. 2006;20(6):670-682

33. Ahmed Z, Mackenzie IR, Hutton ML, Dickson DW Progranulin in frontotemporal lobar degeneration and neuroinflammation. J Neuroinflammation. 2007;4:7.

34. Xu J, et al. Extracellular progranulin protects cortical neurons from toxic insults by activating survival signaling. Neurobiol Aging. 2011;32(12):2326.e5-e16.

35. Cenik B, et al. SAHA (VORINOSTAT) upregulates progranulin transcription: a rational therapeutic approach to frontotemporal dementia. J Biol Chem. 2011;286(18):16101-16108.

36. Capell A, et al. Rescue of progranulin deficiency associated with frontotemporal lobar degeneration by alkalizing reagents and inhibition of vacuolar ATPase. J Neurosi. 2011;31(5):1885-1894. 